\title{
0194 FORENSIC SEXOLOGICAL EXAM IN CHILDREN AND ADOLESCENTS: CHALLENGES OF A PRACTICE
}

M C Maia e Silva, ${ }^{*}$ A De Lima Arajo, L F Santos Souza, S L Barbosa de Souza, A M De Brito Correspondence: Prefeirtura do Recife, Rua Major Codeceira n 194 Santo Amaro, Recife/PE Brasil CEP 50.100-070, CEP 50.100-070, Brazil

10.1136/ip.2010.029215.194

In order to analyze cases of sexual violence, the physical and behavioural signs of which very often go unperceived, 2031 forensic sexology reports at the Institute of Forensic Medicine (city of Recife, northeastern Brazil) on 1144 children and adolescents in 2005 were analyzed. The type of crime for which the exam was requested, forensic evidence and characteristics of the victims and aggressors are presented. Eighty-five per cent of the victims examined were females. Sexual aggression, rape and seduction predominated in $13.4 \%, 28.6 \%$ and $66.7 \%$ of the cases, respectively. Exams for venereal disease were only solicited for 16 victims and were confirmed in six cases. There were differences in exam positivity per type of crime according to age and gender. The main aggressors were neighbour/friend, boyfriend/companion and father. Psychological harm was not was not investigated. The low degree of exam positivity for forensic clarification speaks to the need to adopt protocols and interviewing techniques in order to gain a better recognition of such cases, thereby avoiding re-victimisation and impunity. 